\title{
THE INFLUENCE OF A DIASTASIS SCREW ON THE OUTCOME OF WEBER TYPE-C ANKLE FRACTURES
}

\author{
HUGH R. CHISSELL, JONATHAN JONES
}

From Wexham Park Hospital, Slough, England

We performed a retrospective study of the factors affecting the outcome of Weber type-C ankle fractures in 43 patients reviewed at two to nine years after injury. We determined the functional result in relation to the use of a diastasis screw, the accuracy of reduction, the presence of tibiotalar dislocation, and of injury to the medial side of the ankle by medial malleolar fracture or deltoid ligament rupture. We assessed the use of a diastasis screw as appropriate or inappropriate on the basis of an anatomical study performed by Boden et al (1989).

The diastasis screw was used unnecessarily in 19 of the 31 patients so treated, but this did not appear to affect the final functional result. The worse functional results were in ankles dislocated at the initial injury, and in those with medial malleolar fractures as opposed to those with deltoid ligament ruptures. The best results were after accurate reduction of the fibula and the syndesmosis, and greater increase in the width of the syndesmosis was associated with a worse result. Our results suggest that an increase of more than $1.5 \mathrm{~mm}$ in syndesmosis width is unacceptable.

We recommend that when the deltoid ligament is ruptured, a diastasis screw should be used if the fibular fracture is more than $3.5 \mathrm{~cm}$ above the top of the syndesmosis. When a medial malleolar fracture has been rigidly repaired a diastasis screw is required if the fibular fracture is more than $15 \mathrm{~cm}$ above the syndesmosis.

J Bone Joint Surg /Br/ 1995:77-B:435-8.

Received 20 April 1994: Accepted after revision II Octoher 1994

H. R. Chissell, BSc, FRCS, Senior Orthopaedic Registrar

Charing Cross Hospital, Fulham Palace Road, London W6 8RF. UK.

J. Jones, FRCS, Consultant Orthopaedic Surgeon

Wexham Park Hospital. Slough. Berks SL2 4HL. UK

Correspondence should be sent to $\mathrm{Mr} \mathrm{H}$. R. Chissell at 35 Derby Road. East Sheen, London SW 14 7DU. UK.

(1)1995 British Editorial Society of Bone and Joint Surgery (0301-620X/95/3985\$2.00)
In a Weber type-C ankle fracture (Weber 1966), the fibula is fractured above the level of the joint line usually by a pronation/external rotation force, which causes sequential injury to the medial malleolus or deltoid ligament, the anterior tibiofibular ligaments, the interosseous membrane and the fibula, and finally rupture of the posterior tibiofibular ligament.

Accurate reduction and internal fixation correlate strongly with the final functional result in Weber type-B fractures (Wyss and Zollinger 1991), but the correlation is not so strong for Weber type-C fractures in which other factors such as soft-tissue damage and dislocation also affect the final functional result.

Stability of the syndesmosis after injury was investigated by Boden et al (1989). Their anatomical study on cadaver lower limbs related ankle stability to the height of the fibular fracture above the syndesmosis. They showed that the width of the syndesmosis under constant pronation/ external rotation forces was directly related to the height of the fibular fracture (Fig. 1).

The syndesmosis is more unstable after a high fibular fracture since the interosseus membrane ruptures from the fibular fracture distally. Greater displacement of the syndesmosis is possible when the deltoid ligament has been divided than with a medial malleolar fracture, as the latter can be rigidly fixed to restore stability.

We reviewed our experience with Weber type-C fractures to examine factors which affect the final functional result.

\section{PATIENTS AND METHODS}

We identified 59 patients treated for a Weber type-C ankle fracture at Wexham Park Hospital between 1983 and 1991, and reviewed 43 of them at a mean of 4.5 years ( 2 to 9 ) after the original injury. We used the ankle function score of Karlsson and Peterson (1991), regarding excellent and good functional groups as satisfactory, and fair and poor groups as unsatisfactory. Standard weight-bearing mortice radiographs of both ankles were taken.

The original radiographs were reviewed to record the height of the fibular fracture above the joint line and the nature of the injury to the medial side of the joint. Postoperative radiographs were studied to assess the accuracy of reduction of the fibula and of the syndesmosis; anatom- 


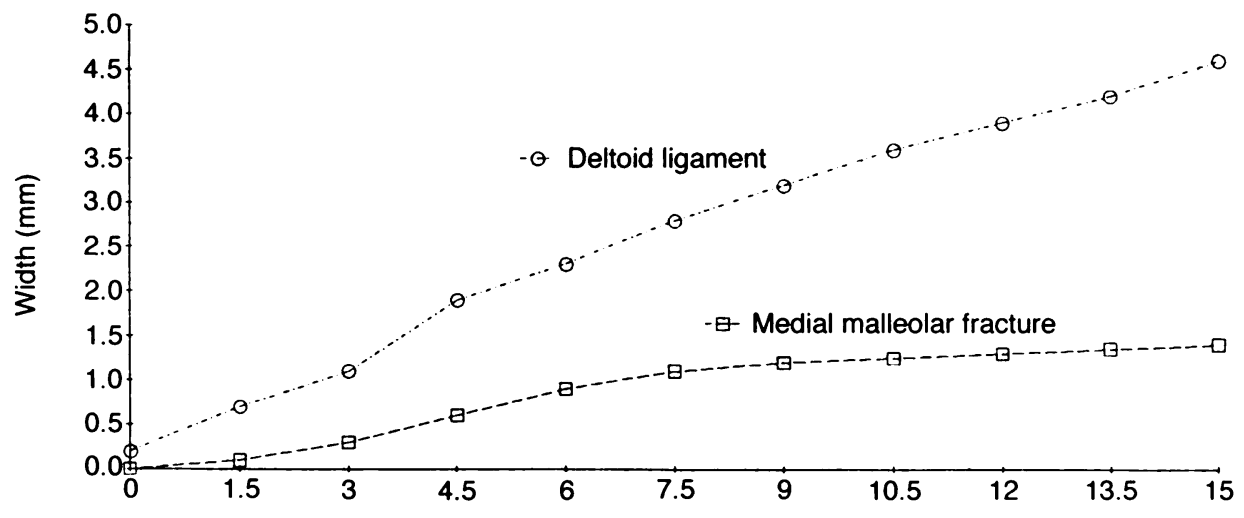

Fig. 1

Increase in width of the syndesmosis related to the height of the fibular fracture in cadaver ankles with either rupture of the deltoid ligament or securely fixed fractures of the medial malleolus (Boden et al 1989).

ical reduction was judged to be satisfactory. On the review radiographs, we measured the width of the syndesmosis at $1 \mathrm{~cm}$ above the ankle joint level as the horizontal distance between the dense line of the tibiofibular notch on the lateral side of the distal tibia and the medial cortical surface of the fibula (Fig. 2).

We then determined the maximum increase in syndesmosis width compatible with a good functional result, by plotting functional results against the increase in width. This figure was applied to Boden's graph to grade the use of diastasis screws as appropriate or inappropriate.

\section{RESULTS}

Functional results. There were 27 satisfactory clinical results (11 excellent, 16 good) and 16 unsatisfactory (11 fair, 5 poor). The mean increase in width of the syndesmosis shown was under $1.5 \mathrm{~mm}$ for all the satisfactory results

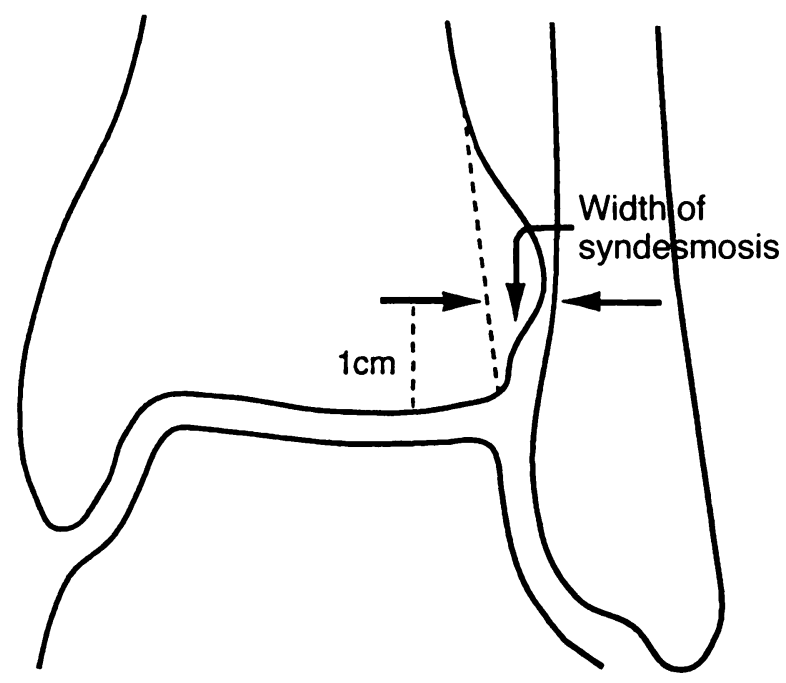

Fig. 2

Method of measuring the width of the syndesmosis (see text).
( $0.8 \mathrm{~mm}$ for excellent, 1.4 for good results) as against $2 \mathrm{~mm}$ for the fair and $3.8 \mathrm{~mm}$ for the poor results. Boden's graph showed that an increase of width of $1.5 \mathrm{~mm}$ corresponded to a fibular fracture at $3.5 \mathrm{~cm}$ above the top of the syndesmosis in an ankle with a deltoid ligament rupture and over $15 \mathrm{~cm}$ in an ankle with a rigidly fixed medial malleolar fracture.

Use of diastasis screws. A diastasis screw had been used in 31 of our 43 patients. Of the 12 with no screw four were managed conservatively and eight by operation without a diastasis screw. The omission of a diastasis screw was appropriate in all eight. In the 31 patients having diastasis screws, 19 screws were considered inappropriate, most because rigid fixation of a medial malleolar fracture had given adequate stability. We found no statistically significant difference between the functional results of appropriate or inappropriate use of a diastasis screw (Fig. 3).

Medial malleolar fracture or deltoid ligament rupture. After a medial malleolar fracture there was only one excellent result, but after rupture of the deltoid ligament there were ten excellent results (Fig. 4). The difference in results was significant (chi-squared test; $\mathrm{p}<0.05$ ).

Tibiotalar dislocation. Only four of the 27 patients with satisfactory results had presented with a fracture dislocation of the ankle, and all four had only good results. By contrast, seven of the 16 patients with unsatisfactory results had fracture dislocations and these included four of the five patients who had poor functional results (chi-squared test; $\mathrm{p}<0.05$; Fig. 5).

Reduction of the syndesmosis. Eighteen of the 23 patients $(78 \%)$ with satisfactory results had an anatomical reduction of the syndesmosis compared with nine of the $16(56 \%)$ with unsatisfactory results. This difference was not statistically significant (Fig. 6).

\section{DISCUSSION}

The main stabilising feature of the ankle mortice is the interosseus membrane (Riegels-Nielsen, Christensen and 


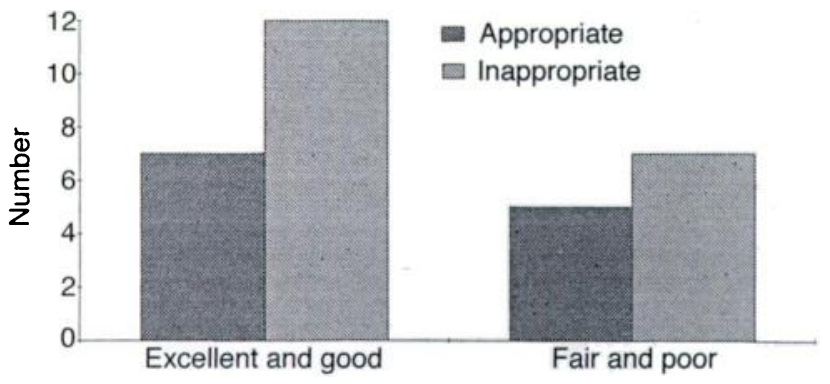

Fig. 3

Appropriate and inappropriate use of a diastasis screw related to the functional result.

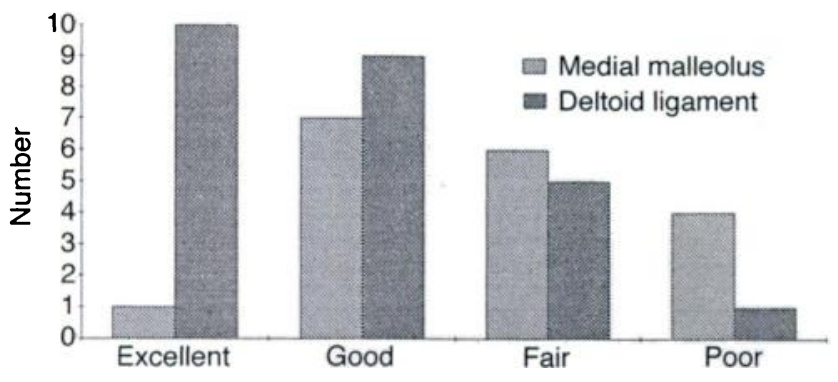

Fig. 4

Associated deltoid ligament injury or fixed medial malleolar fracture related to the functional result.

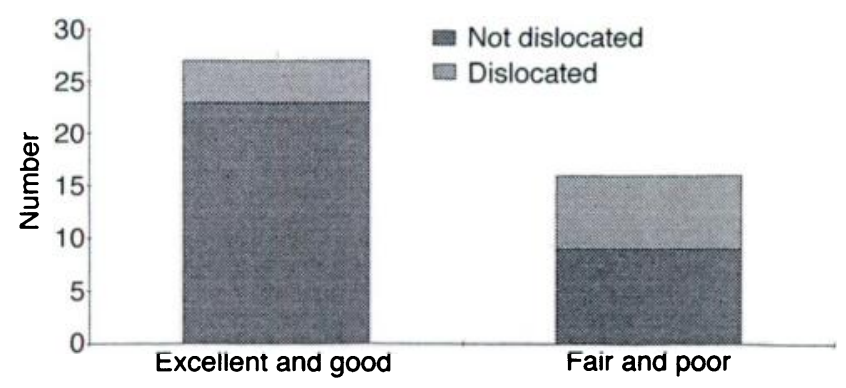

Fig. 5

Tibiotalar dislocation related to the functional result.

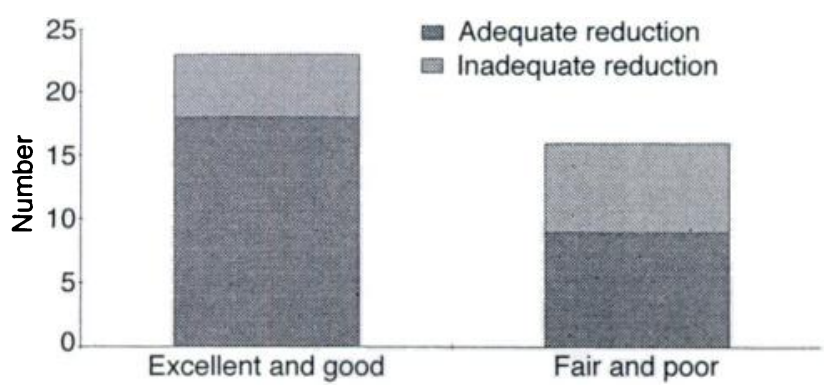

Fig. 6

Reduction of the syndesmosis related to the functional result.
Greiff 1983). In a Weber type-C ankle fracture this is ruptured below the most distal extension of the fibular fracture (Roberts 1983). Experimental studies of these fractures have shown that rigid repair of the injury to the medial side alone restores more stability than any other single factor and that this is augmented by rigid fixation of the fibular fracture. Rigid repair of the fibular fracture alone provides less stability and in such a case the addition of a syndesmosis screw may be required to achieve stability. An ankle with an unrepaired injury of the deltoid ligament is therefore considerably less stable than that with a fixed medial malleolar fracture, and a diastasis screw will be required if the fibular fracture is more than a few centimetres from the joint line (Boden et al 1989; Solari et al 1991).

The height of a fracture which will require a diastasis screw depends upon the maximum increase in the width of the syndesmosis which is compatible with satisfactory function. This has been reported to vary from $1 \mathrm{~mm}$ to $2 \mathrm{~mm}$ (Pettrone et al 1983; Leeds and Ehrlich 1984; Lindsjo 1985), and our results indicate that the maximum permissible increase is $1.5 \mathrm{~mm}$.

In patients with deltoid ligament ruptures this level of increase is seen when the fibular fracture is $3.5 \mathrm{~cm}$ above the syndesmosis. A diastasis screw should be used for fractures proximal to this. After rigid repair of a medial malleolus the syndesmosis is more stable and a diastasis screw is required only for fractures of the proximal fibula which are more than $15 \mathrm{~cm}$ above the joint line.

In some pronation/external rotation injuries, the posterior band of the inferior tibiofibular ligament may remain intact. A diastasis screw will not then be required for some high fractures. Stressing the syndesmosis at the time of operation will help to determine this and may prevent unnecessary use of a diastasis screw.

In our series over $60 \%$ of the diastasis screws were used unnecessarily, since fixation of the malleoli had already provided adequate stability. The final functional result was probably not affected by this but short-term morbidity was increased because unprotected weight-bearing was delayed and the screw required removal.

A diastasis screw prevents normal movement between the tibia and fibula, and may encourage the development of a synostosis (Kaye 1989). Weight-bearing with a diastasis screw in place may cause the screw to break or loosen with the appearance of a lucent line around the screw in the tibia (Kaye 1989; Stiehl and Schwartz 1990).

We found that patients who had sustained a concomitant medial malleolar fracture had worse functional results and this was also reported by Colton (1971). Broos and Bisschop (1991) considered that this difference was due to damage to the articular surface of the tibial plafond at the time of injury.

Dislocation of the tibiotalar joint at the time of the initial injury gave a significantly worse functional result, probably due to articular cartilage damage at the time of dislocation 
(Hughes et al 1979). Two of our five patients with poor results had been seen to have damage to the articular cartilage of the dome of the talus at the time of operation. Dislocation must also cause more soft-tissue damage

Anatomical reduction improves the final result and reduces the subsequent incidence of osteoarthritis (Pettrone et al 1983; Roberts 1983; Leeds and Ehrlich 1984; Lindsjo 1985; Stiehl and Schwartz 1990). Degenerative arthritis has been shown to occur much sooner after Weber type-C than Weber type-B fractures despite anatomical reduction (Wyss and Zollinger 1991), and the less accurate reduction achieved by conservative treatment also leads to an increased incidence of arthritis in late radiological follow-up (Stiehl and Schwartz 1990). This may require arthrodesis much earlier than similar fractures managed operatively (Wyss and Zollinger 1991)

Conclusions. Diastasis screws are probably overused: this increases short-term morbidity, but has little effect on longterm functional results. Anatomical reduction of the fibula is essential.

Association with medial malleolar fractures gives worse functional results than deltoid ligament ruptures, probably due to damage to the mortice at the time of injury.

A diastasis screw should be used for fibular fractures more than $3.5 \mathrm{~cm}$ above the joint level if the deltoid ligament is ruptured and $15 \mathrm{~cm}$ above if there has been a medial malleolar fracture. The mortice should be stressed after fixation of the malleoli and a diastasis screw used if there is significant laxity.

No benefits in any form have been received or will be received from a commercial party related directly or indirectly to the subject of this article.

\section{REFERENCES}

Boden SD, Labropoulos PA, McCowin P, Lestini WF, Hurwitz SR. Mechanical considerations for the syndesmosis screw: a cadaver study. J Bone Joint Surg [Am] 1989;71-A:1548-55.

Broos PL, Bisschop PLG. Operative treatment of ankle fractures in adults: correlation between types of fracture and final results. Injury 1991:22:403-6.

Colton CL. The treatment of Dupuytren's fracture-dislocation of the ankle. J Bone Joint Surg [Br] 1971:53-B:63-71.

Hughes JL, Weber H, Willenegger H, Kuner EH. Evaluation of ankle fractures: non-operative and operative treatment. Clin Orthop 1979;138:111-9.

Karlsson J, Peterson L. Evaluation of ankle joint function: the use of a scoring scale. The Foot 1991:1:15-9.

Kaye RA. Stabilization of ankle syndesmosis injuries with a syndesmosis screw. Foot and Ankle 1989:9:290-3.

Leeds HC, Ehrlich MG. Instability of the distal tibiofibular syndesmosis after bimalleolar and trimalleolar ankle fractures. J Bone Joint Surg [Am] 1984;66-A:490-503.

Lindsjo U. Operative treatment of ankle fracture dislocations: a follow-up study of 306/321 consecutive cases. Clin Orthop 1985;199:28-38.

Pettrone FA, Gail M, Pee D, Fitzpatrick T, Van Herpe LB. Quantitative criteria for prediction of the results after displaced fracture of the ankle. J Bone Joint Surg [Am] 1983;85-A:667-77.

Riegels-Nielsen P, Christensen J, Greiff J. The stability of the tibiofibular syndesmosis following rigid internal fixation for type $\mathrm{C}$ malleolar fractures: an experimental and clinical study. Injury 1983:14:357-60.

Roberts RS. Surgical treatment of displaced ankle fractures. Clin Orthop 1983:172:164-70

Solari J, Benjamin J, Wilson J, Lee R, Pitt M. Ankle mortise stability in Weber C fractures: indications for syndesmotic fixation. J Orthop Trauma 1991:5:190-5.

Stiehl JB, Schwartz HS. Long-term results of pronation-external rotation ankle fracture-dislocations treated with anatomical open reduction, internal fixation. J Orthop Trauma 1990;4:339-45.

Weber BG. Die Verletzungen des oberen Springgelenkes. In: Aktuelle Probleme in der Chirurgie. Bd 3. Berne and Stuttgart; Huber, 1966.

Wyss $\mathbf{C}$, Zollinger $\mathbf{H}$. The causes of subsequent arthrodesis of the ankle joint. Acta Orthop Belg 1991;Suppl 57:22-7. 